Draft VERSiOn MAY 10, 2019

Preprint typeset using $\mathrm{LAT}_{\mathrm{E}} \mathrm{X}$ style emulateapj v. 5/2/11

\title{
OBSERVATION OF THE EVOLUTION OF A CURRENT SHEET IN A SOLAR FLARE
}

\author{
Chunming Zhu ${ }^{1}$, Rui Liu ${ }^{2,3}$, David Alexander ${ }^{4}$, R.T. James McAteer ${ }^{1}$ \\ ${ }^{1}$ Department of Astronomy, New Mexico State University, NM 88003, USA; czhu@nmsu.edu \\ ${ }^{2}$ CAS Key Laboratory of Geospace Environment, Department of Geophysics and Planetary Sciences, University of Science and \\ Technology of China, Hefei 230026, China \\ ${ }^{3}$ Collaborative Innovation Center of Astronautical Science and Technology, China and \\ ${ }^{4}$ Department of Physics and Astronomy, Rice University, TX 77005, USA \\ Draft version May 10, 2019
}

\begin{abstract}
We report multi-wavelength and multi-viewpoint observations of a solar eruptive event which involves loop-loop interactions. During a C2.0 flare, motions associated with inflowing and outflowing plasma provide evidence for ongoing magnetic reconnection. The flare loop top and a rising "concaveup" feature are connected by a current-sheet-like structure (CSLS). The physical properties (thickness, length, temperature, and density) of the CSLS are evaluated. In regions adjacent to the CSLS, the EUV emission (characteristic temperature at 1.6 MK) begins to increase more than ten minutes prior to the onset of the flare, and steeply decreases during the decay phase. The reduction of the emission resembles that expected from coronal dimming. The dynamics of this event imply a magnetic reconnection rate in the range $0.01-0.05$.
\end{abstract}

Subject headings: Sun: corona - Sun: flares

\section{INTRODUCTION}

Magnetic reconnection, a physical process involving the topological reconfiguration of magnetic fields, is generally accepted as a key mechanism for the release of free magnetic energy during solar eruptive events. Many observational features of magnetic reconnection in flares have been reported, including cusp-shaped flare loops (Tsuneta et al. 1992), above-the-loop-top hard X-ray sources (Masuda et al. 1994), plasma inflows toward the reconnection site (e.g., Yokoyama et al. 2001; Liu et al. 2010; Su et al. 2013), plasma outflows (e.g., Wang et al. 2007; Tian et al. 2014) and their associated plasma blob ejections (Takasao et al. 2012) and loop shrinkage, i.e. retraction of newly reconnected field lines from the reconnection site ( Švestka et al. 1987; McKenzie \& Hudson 1999; Savage \& McKenzie 2011; Liu et al. 2013).

During magnetic reconnection, current sheets are expected to form at the neutral region of the converging anti-parallel magnetic fields. Several observations of CSLS were reported from X-ray and EUV images of solar flares (e.g., Sui \& Holman 2003; Savage et al. 2010; Liu et al. 2010) and white light coronagraph observations of coronal mass ejections (CMEs) (Ko et al. 2003; Webb et al. 2003; Lin et al. 2007; Ciaravella \& Raymond 2008). However, the physical properties and the evolution of the current sheet during a solar flare still remain unclear (see the recent review by Lin et al. 2015). Further, although the standard 2D flare model (e.g., Kopp \& Pneuman 1976) successfully explains several observed features during flares, the solar flare is intrinsically a 3D phenomenon (Su et al. 2013; Sun et al. 2015). In this study, we present the evolution of a CSLS in a solar flare observed on 2013 December 10 in multiple wavelengths from multiple viewpoints. The physical properties of the flaring structures and their associated dynamics are quantified. From these dynamics, we estimate the magnetic reconnection rate for this event. We also discuss the possible implications of these structures for our understanding of loop interactions, and associated reconnection, in solar flares.

\section{OBSERVATIONS}

\subsection{Instruments}

The flare under study occurred on 2013 December 10 in AR 11916 at location of W60S15. We report the observations provided by three spacecraft from two different viewing angles (Figure 1(d)), i.e. the Solar Dynamics Observatory (SDO; Pesnell et al. 2012) and Hinode (Kosugi et al. 2007) near the Earth and the Solar Terrestrial Relations Observatory (STEREO; Kaiser et al. 2008) Ahead spacecraft (STEREO-A), with a separation angle of around 150 degrees. The Atmospheric Imaging Assembly (AIA; Lemen et al. 2012) aboard SDO takes fulldisk images of the Sun in $10 \mathrm{EUV} / \mathrm{UV}$ channels $(\log (T)$ ranges $3.7-7.3)$, with roughly 12 -second cadence. Fulldisk magnetograms are provided by the Helioseismic and Magnetic Imager (HMI; Schou et al. 2012) on board SDO, with $1^{\prime \prime}$ spatial resolution and 45-second cadence. The X-ray Telescope (XRT; Golub et al. 2007) on board Hinode provides complementary observations of this active region in multiple bandpasses with a scale of $\sim 1^{\prime \prime}$ pixel $^{-1}$. This flare appears at the eastern limb from the perspective of STEREO-A. The Extreme-Ultraviolet Imager (EUVI; Wuelser et al. 2004) on board STEREO observed the Sun in four bandpasses, with a scale of $1.6^{\prime \prime}$ pixel $^{-1}$ and a cadence of 5 minutes in the $195 \AA$ filter.

\subsection{Results}

The X-ray peak of the flare was recorded at $~ 07: 47$ UT by the Geostationary Operational Environmental Satellite $(G O E S)$ with a magnitude of $\mathrm{C} 2.0$. Figure 1(f) shows both the evolution of the GOES X-ray emission and the associated light curve in AIA $131 \AA(\sim 10 \mathrm{MK})$ covering this flare region.

Starting at $~ 06: 54$ UT, several short hot loops (SHL) (Figure 2(a)) visible in AIA $131 \AA$, erupted westward 

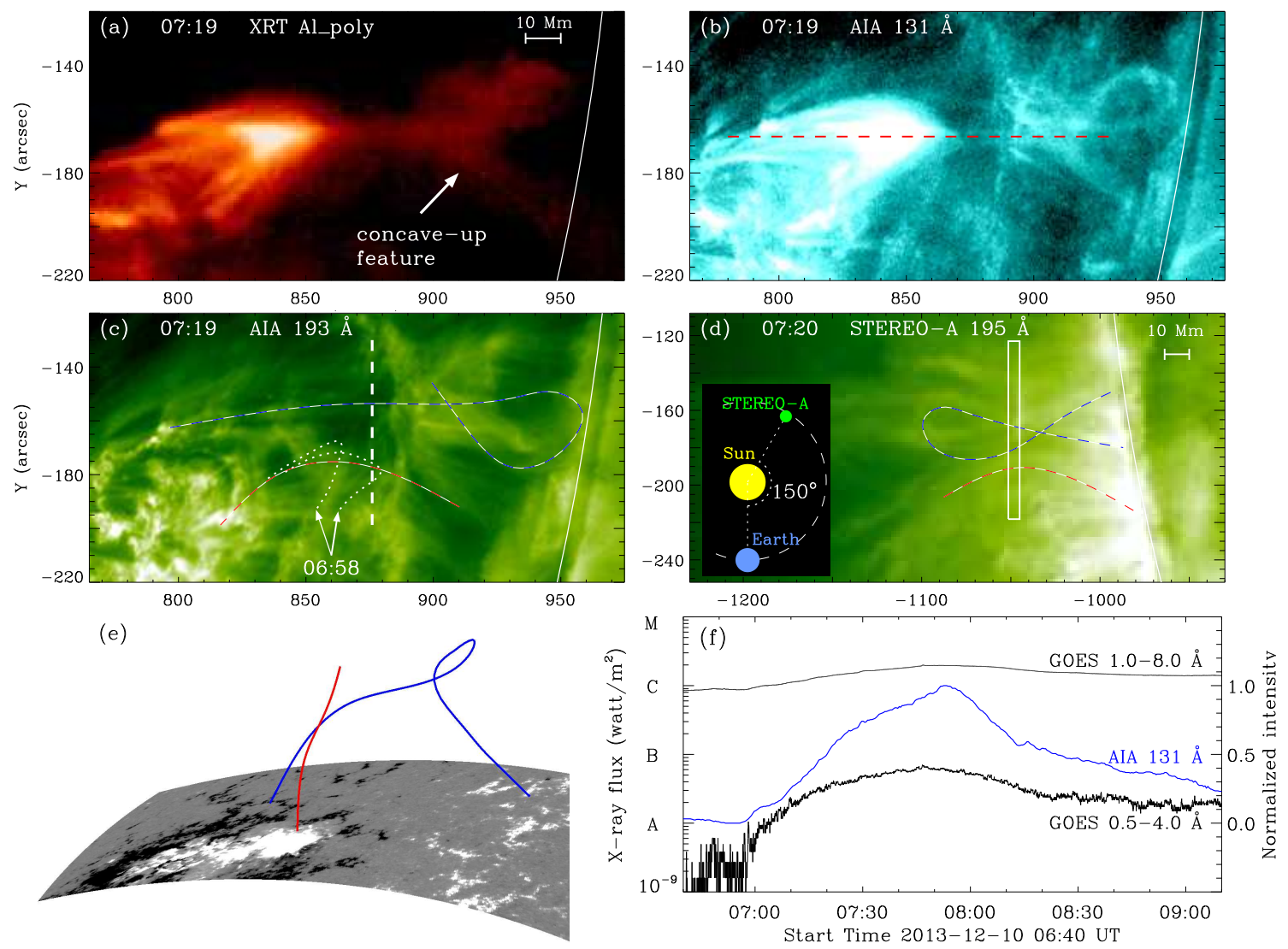

FIG. 1.- A solar flare and associated structures observed from Hinode, SDO, and STEREO-A. A horizontal slit in (b) is selected to study the outward motions. A vertical slit in (c) and another 5-pixels-width slit in (d) are chosen to study the inward motions for SDO/AIA and STEREO-A/EUVI, respectively. Profiles of two short loops in the south at 06:58 UT are marked with dotted lines. The inset of (d) displays the relative locations of the spacecraft. Two typical converging groups of long loops are marked by the dashed lines in (c) and (d). Their 3D reconstruction is shown in (e). The grey-scaled surface displays the differentially-rotated photospheric magnetic fields observed two days before this flare. This approach is chosen to reduce the projection effects due to the location of this active region near the west limb. (f) The light curves of GOES and normalized intensity of AIA $131 \AA$.

( Animations of this figure are available, see Animations 1 and 2.)

(or upward) and away from the flare loop-top region. A few of them appear as sharply-angled structures (Figure $2(\mathrm{a} 1))$ probably due to projection of the tilted loops. Meanwhile, the nearby loops observed in $193 \AA(\sim 1.6$ MK) immediately to the north and south of this region converged, see Figure 1(c) and Animation 1. The loops observed in the north were noticeably kinked, see Figures $1(\mathrm{~b})$ and (c). In the south, both short and long loops are observed, marked in Figure 1(c). The 3D configurations of two groups of converging long loops (dashed lines in Figures 1(c) and (d)) are reconstructed using the SolarSoft routine scc_measure (Thompson et al. 2012), see Figure 1(e) and Animation 2. The northern kinked loop had its eastern and western footpoints connected to the dispersed negative and positive polarities on the solar surface, respectively. The southern long loop had one root in the condensed positive polarity and deviated southward (the other part of the southern loop is not included here due to its strong background emission). These configurations suggest that these loops are not coplanar.

At 07:08 UT, a "V-shaped" concave-up feature (CUF) appeared in both XRT X-rays ("Thin Al_poly" filter, $\sim 2-10$ MK, see Figures 1(a)) and AIA $131 \AA$ (see
Figures 1(b)). The development of this feature is clearly shown in Animation 1. Similar features can be found in the previous observations of loop-loop interaction ( $\mathrm{Su}$ et al. 2013) and plasmoid ejections (Liu et al. 2013). The CUF was connected to the flare loop-top region by a thin layer. The location of this thin layer, and its appearance in the hot channels, suggest it is a CSLS.

An erupting dark loop, best identified through a series of running difference images (Figure 2(e)), was detected at the west solar limb at 07:11 UT and appeared as a relatively dim structure in AIA $171 \AA(\sim 0.6 \mathrm{MK}$, see Animation 3$)$. Its location and velocity $\left(\sim 60 \mathrm{~km} \mathrm{~s}^{-1}\right)$ indicate this dark loop might correspond to an SHL that reached the solar limb. No obvious CME is evident in $\mathrm{SOHO} / \mathrm{LASCO}$ data to be associated with the erupting loop structures, possibly because they were too faint at higher altitudes.

A north-south virtual slit in Figure 1(c) is selected to generate a space-time stackplot (Figures $3(\mathrm{a})$ and (b)). Several inward flows are indicated as converging streaks, with velocities derived by linear fittings and shown in Figure 3(d). Inward velocities have an average value of $\sim 10 \mathrm{~km} \mathrm{~s}^{-1}$, and tend to increase as they approached the CSLS, similar to the observation by Su et al. (2013). 

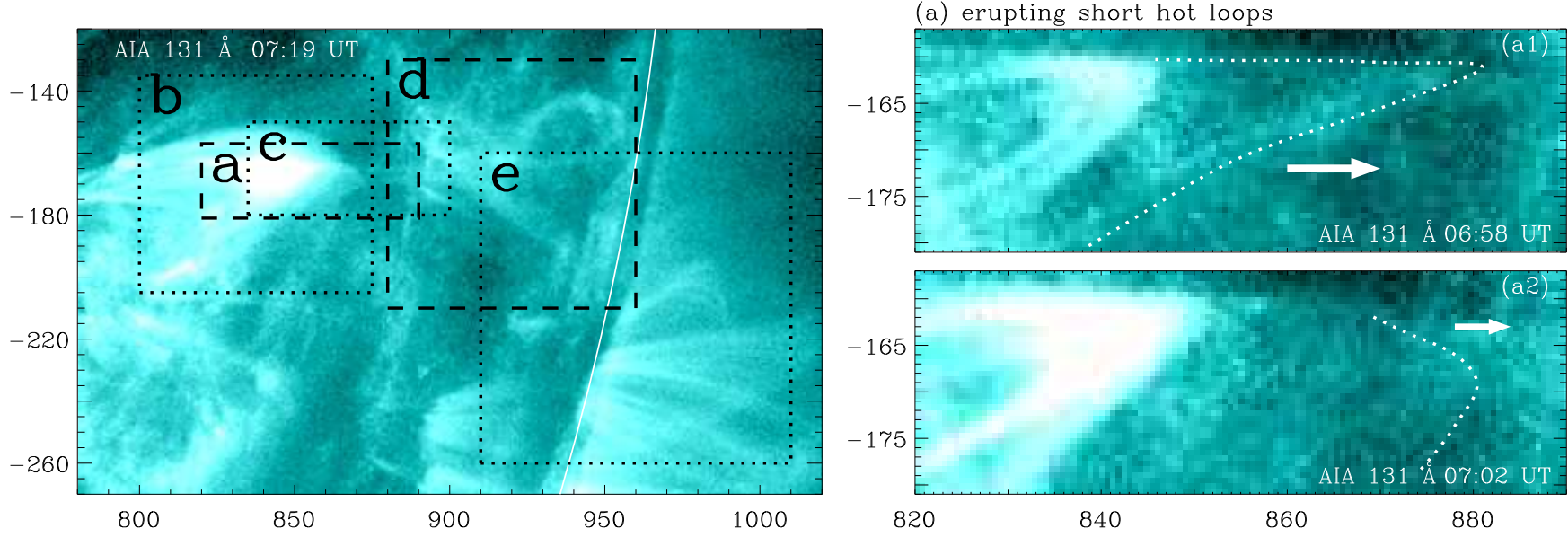

(b) loop shrinkages

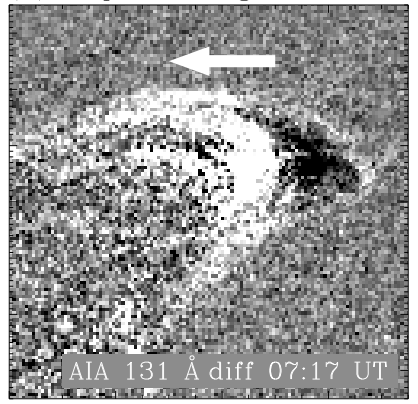

(c) a blob-like structure

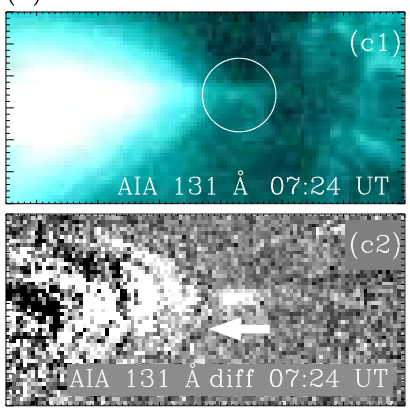

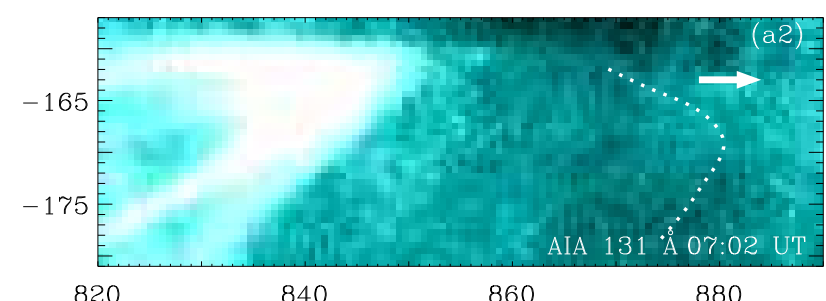

(d) ejecting loops at CUF

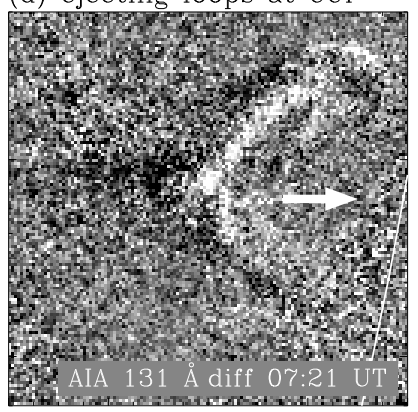

(e) an erupting dark loop

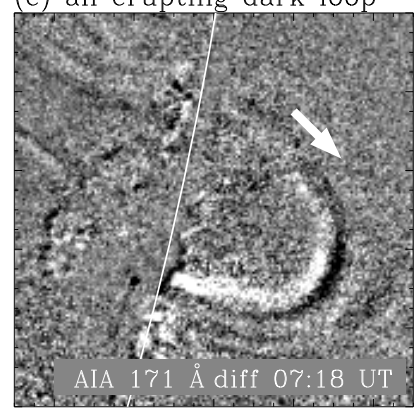

FIG. 2. - Several moving features observed during the solar flare. The relative positions are displayed in the top left panel. Each box is labeled to match the corresponding panels. (a) Erupting short hot loops (SHL). (b) A running difference image showing downward loop shrinkages. (c) A blob-like structure in AIA 131 A (c1) and a running difference image (c2). (d) Upward ejecting loops at the CUF. (e) A running difference image displaying an erupting dark loop beyond the solar limb. The arrow in each case indicates the direction of motion. (Animations of this figure is available, see Animations 3 and 4.)

Two groups of loops in the north observed between 07:10 and 07:25 UT show relatively larger final velocities of $\sim 30 \mathrm{~km} \mathrm{~s}^{-1}$. Similar trajectories are also observed from STEREO-A in EUVI $195 \AA$, see Figure 3(c), generated along a slit marked in Figure 1(d).

Motions along the CSLS are studied with an east west oriented slit, see Figure 1(b). The trajectories of four moving features are presented in Figure 3(e), including the erupting SHL, shrinking loops at the flare apex, upward ejecting loops at the CUF, and blob-like structures (Figure 2). 1) A series of erupting SHL (Figure 2(a)) primarily observed in the early stage of the flare from 06:54 UT to 07:12 UT, exhibit velocities ranging from 45 to $240 \mathrm{~km} \mathrm{~s}^{-1}$ (Figure $3(\mathrm{f})$ ), with an average value of $\sim 150 \mathrm{~km} \mathrm{~s}^{-1}$. 2) The downward shrinking loops (Figure 2(b)), which initiated simultaneously with the erupting SHL, retract continuously towards the loop-top region until $\sim 08: 10 \mathrm{UT}$. The velocities of these shrinking loops changed from between $\sim 40-80 \mathrm{~km} \mathrm{~s}^{-1}$ to less than $10 \mathrm{~km} \mathrm{~s}^{-1}$ in $\sim 10$ minutes. 3) Several upward ejecting loops appear successively (one example is shown in Figure $2(\mathrm{~d})$ ) along with the presence of the CUF, with average initial velocities of $\sim 160 \mathrm{~km} \mathrm{~s}^{-1}$, significantly faster than the downward ones. This phenomenon is consistent with previous observations by Liu et al. (2013) and is thought to be related to higher density near the flare top, which can strongly decelerate downward flows. 4) Between 07:22 and 07:28 UT, two blob-like structures (with the first one shown in Figure 2(c)) moved downward with velocities of approximately 135 and $143 \mathrm{~km} \mathrm{~s}^{-1}$, respectively. These values are typically found in blobs (see Shen et al. (2011) and references therein).

The projected dimensions of the CSLS are studied at 07:19 UT, by which time it was fully developed, see Figure 4. The normalized intensities along a vertical slit in AIA $131 \AA$ (Figures 4(a)) are shown in Figure 4(c). A Gaussian fitting applied to this profile gives a full-widthat-half-maximum (FWHM) value of $5.3 \operatorname{arcsec}(3.8 \mathrm{Mm})$. The apparent thickness is necessarily an upper limit because the presence of several factors: 1) projection effects on the width of the current sheet (Lin et al. 2009), 2) a possible "thermal halo" around it (e.g., Yokoyama \& Shibata 2001), 3) the 3D shape of the current sheet (Fan \& Gibson 2007), and 4) projection of nearby hot loops onto the CSLS region. However, the value attained agrees well with past observations, such as $5-10 \mathrm{Mm}$ in Liu et al. (2010), and 4-5 Mm in Savage et al. (2010).

The intensities of the X-rays along the CSLS display two localized maxima: a strong source at the flare loop top, and a much weaker source at the CUF, see Figure $4(\mathrm{~d})$. The length of the CSLS is estimated by measuring the distance between the two X-ray maxima. The evolution of this length is displayed in the inset of Figure 4(d). The CSLS extended from $60.7 \operatorname{arcsec}(44 \mathrm{Mm})$ at 07:05 UT to $79.2 \operatorname{arcsec}(57 \mathrm{Mm})$ at 07:25 UT, increasing with a velocity of $10.7 \mathrm{~km} \mathrm{~s}^{-1}$ given by a linear 

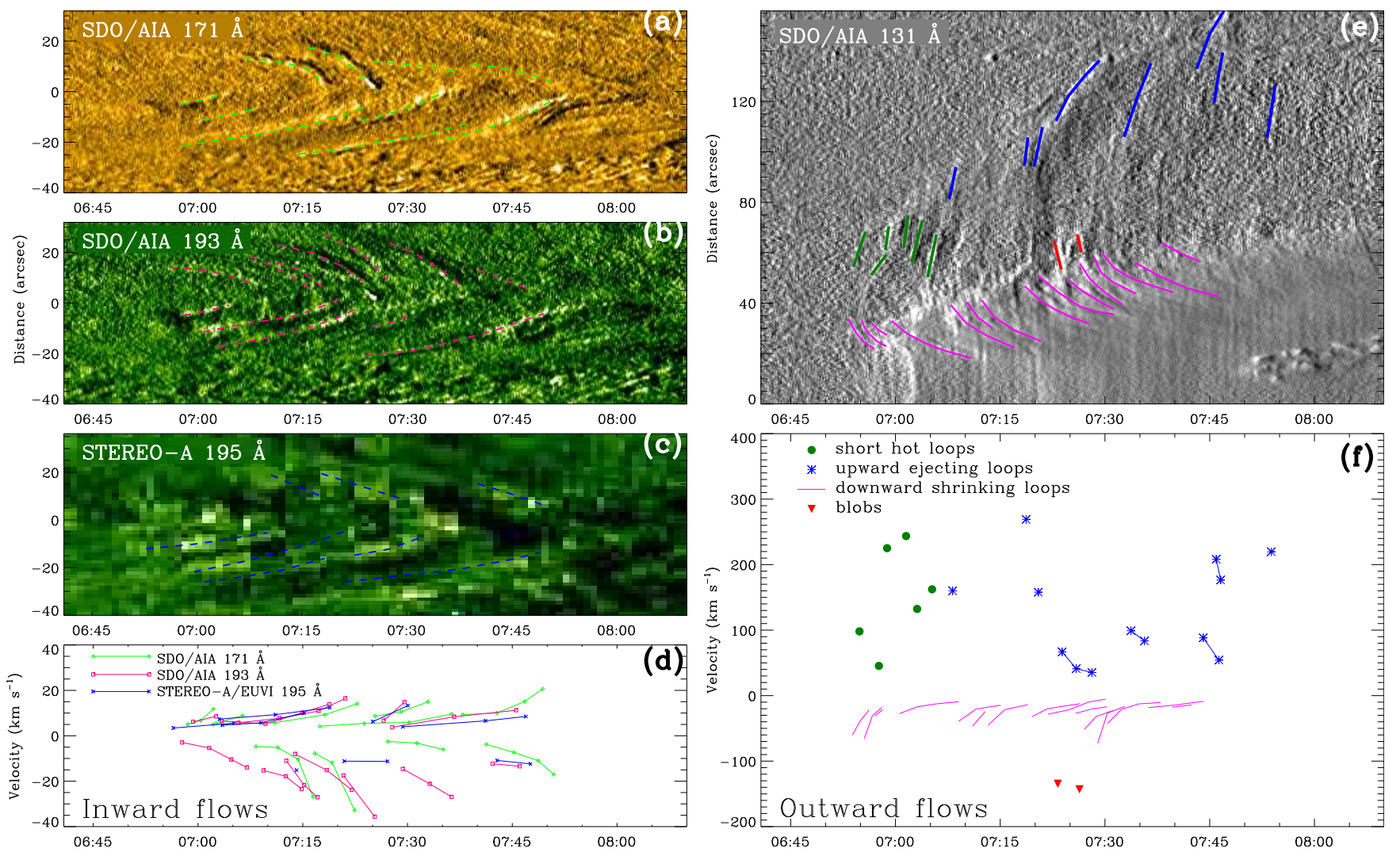

FIG. 3.- Inward and outward motions. The converging flows along the vertical slit in Figure 1(c) are displayed in a running ratio difference of the images, with (a) from SDO/AIA $171 \AA$, (b) from SDO/AIA $193 \AA$, and (c) from STEREO-A $195 \AA$, denoted by the dashed curves. Velocities are shown in (d). (e) A stackplot along the horizontal slit marked in Figure 1(b). The green lines denote the erupting short hot loops, the red lines for the blobs. The pink lines mark the downward shrinking loops. The blue lines mark the upward ejecting loops at the CUF. (f) Velocities of the outward motions identified in (e).

(Animations of this figure is available, see Animations 3 and 4.)

fit, and indicated by a dashed line. As the flare progresses, the thin layer rises and extends until it is barely detectable after 07:50 UT. With the estimated length $L$ $\sim 50 \mathrm{Mm}$, and thickness $d \sim 4 \mathrm{Mm}$, the measured area of the CSLS, $A_{\mathrm{cs}}$, is $\sim 200 \mathrm{Mm}^{2}$.

The intensity of emission in AIA $193 \AA$ around the CSLS varies as the flare progresses. A stackplot along the north-south slit (Figure 1(c)) between 06:00 - 09:18 UT is displayed in Figure 4(e). The white curve (used as "Lightcurve 193" in the following) denotes the normalized total intensities of the vertical pixels within \pm 27 arcsec (dashed lines) neighboring the CSLS. It displays three phases, denoted by $\mathrm{P} 0-\mathrm{P} 2$, respectively. 1) P0 (06:00 - 06:28 UT): Lightcurve 193, and that from GOES (marked by a black curve), are almost flat during this phase, providing a background level in this region. 2) P1 (06:28-07:58 UT): The intensity is larger than the background. It is interesting to note that Lightcurve 193 begins to increase more than 10 minutes earlier than that of GOES. Without noticeable changes in the background (see Animation 5), the increased emissions were probably caused by two factors: i) brightenings of the loops within this region, such as those marked by an arrow in Figure 4(e), that become more evident in Phase P1; and ii) brighter material/loops moving into this region. Lightcurve 193 peaks during Phase 1 at 07:10 UT and then decreases. 3) P3 (07:58 - 09:18 UT): Lightcurve 193 falls below the levels of $\mathrm{P} 0$ and $\mathrm{P} 1$, indicating reduced emission. A relatively steep reduction appears between 07:58 - 08:10 UT, during the decay phase of the flare. The lower emission might be related to coronal dimming, as reported by previous observations (e.g., Sterling \& Hudson 1997; Zarro et al. 1999 and see references therein) and the simulation by Reeves et al. (2010).

A comparison of the normalized average intensities of the horizontal pixels during each of the phases, P0 - P2, is shown in Figure 4(f). 1) Within 27 arcsec south of the CSLS, it is clear that the intensities in P1 $>\mathrm{P} 0$. At the same time, and within the same range of the northern portion of the flaring region, $\mathrm{P} 1$ has lower intensity than P0 near the CSLS and higher intensity than P0 further from the CSLS. These variations suggest that the increased intensity during $\mathrm{P} 1$ originated primarily from the southern side. 2) The intensities on both sides of the CSLS during P2, compared with P0 and P1, significantly decreased. This reduction of the EUV emission adjacent to the CSLS is consistent with the numerical study of coronal dimming (Reeves et al. 2010). 3) The southern side of the CSLS tends to be brighter than the north, probably due to the line-of-sight integration effect of the emissions from distinct coronal loops, or different temperatures and/or densities of the loops neighboring the CSLS.

The plasma properties of the structures are studied 

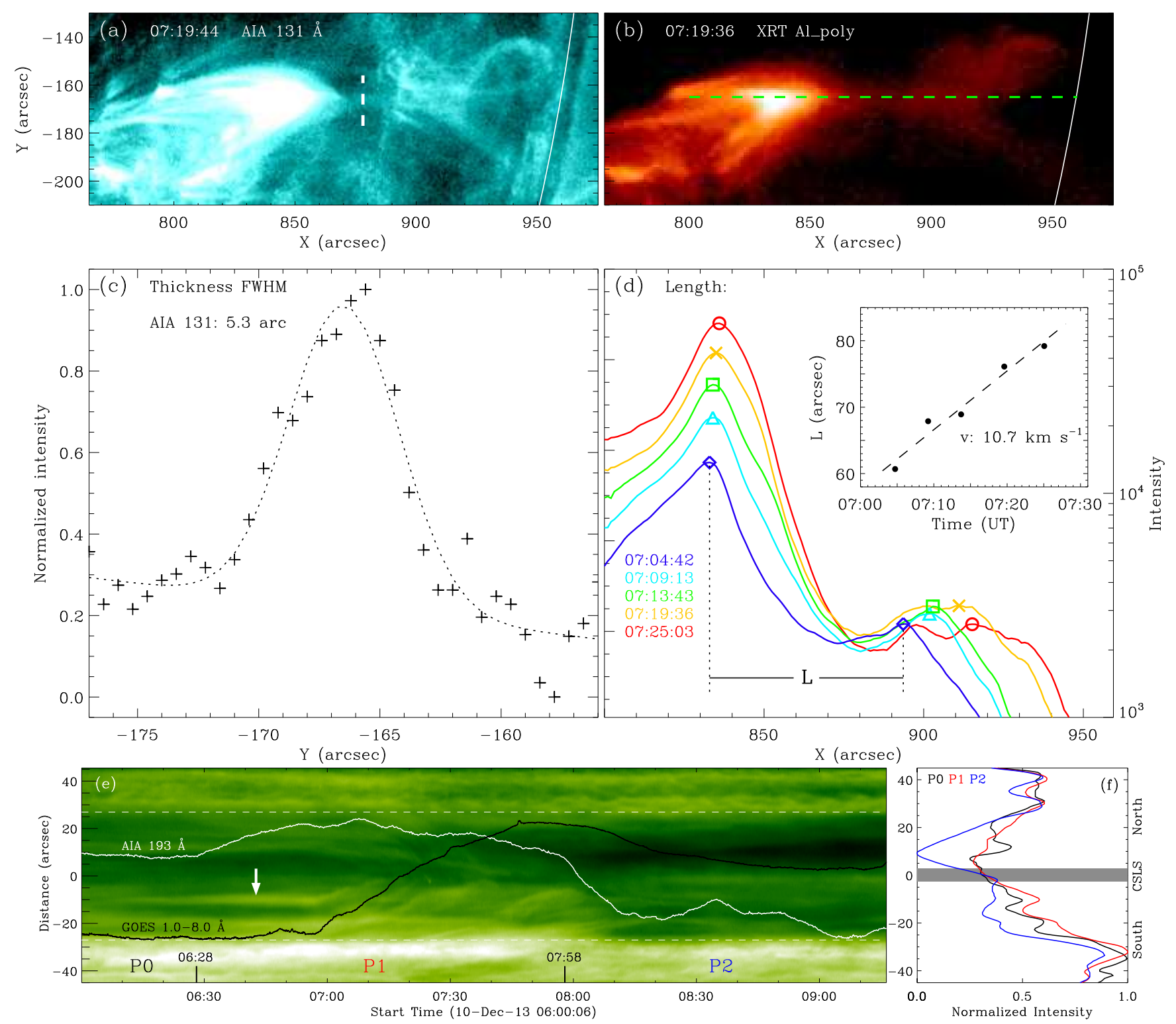

FIG. 4. - The thickness and length of the CSLS, and the nearby variation of the emission. (a) AIA $131 \AA$ image at 07:19 UT. (b) XRT Al_poly filtered image at the same time. (c) Normalized intensity along the vertical slit in (a), with Gaussian fit marked by the dotted curve. The Full width at half maximum (FWHM) of the Gaussian fit is 5.3 arcsec. (d) Intensities along the horizontal slit marked in (b). The length of the CSLS, denoted by the distance between two localized maxima (marked by the same symbols) along each smoothed curve at five times, indicating an ongoing extension of the CSLS. A linear fitting to the growth in length is displayed in the inset. (e) Stackplot along the vertical slit in 1 (c). The white curve marks the normalized total intensities of the vertical pixels between the two dashed lines. It displays three phases of P0-P2. The black curve denotes the lightcurve of GOES X-rays. A region with increasing emission is denoted by the arrow. (f) Normalized average values of the horizontal pixels within the three phases. The grey region, with a width of 5.3 arcsec, denotes the CSLS.

(Animations of this figure is available, see Animations 1 and 5.)

with a regularization method using AIA images to determine differential emission measures (DEMs; Hannah \& Kontar 2012). The DEM profiles are investigated in three brightening regions (R1 - R3) associated with the flaring structures and a neighboring reference box R0, see Figure 5(a). They all display a bimodal distribution: a cold component peaking at $\sim 2 \mathrm{MK}$, and a hot component ranging from $7-16 \mathrm{MK}$. The cold components from the flaring regions R1 - R3 have similar DEM values to the reference region $\mathrm{R} 0$, suggesting this component probably came from the foreground and background in line with the flaring region (see Battaglia \& Kontar 2012). The hot components, however, varied among the regions. This suggests that the hot components are associated with the flare and that they have temperatures of 7 $16 \mathrm{MK}$. The appearance of the hot component at R0 is likely an artifact due to the SDO/AIA response calibration (e.g., Battaglia \& Kontar 2012) or may be related to the apparently prevalent hot plasma in the solar corona (Schmelz et al. 2009). Another issue with the regularization method of AIA images is that it is limited to a temperature up to $\sim 18 \mathrm{MK}$, corresponding to the for- 

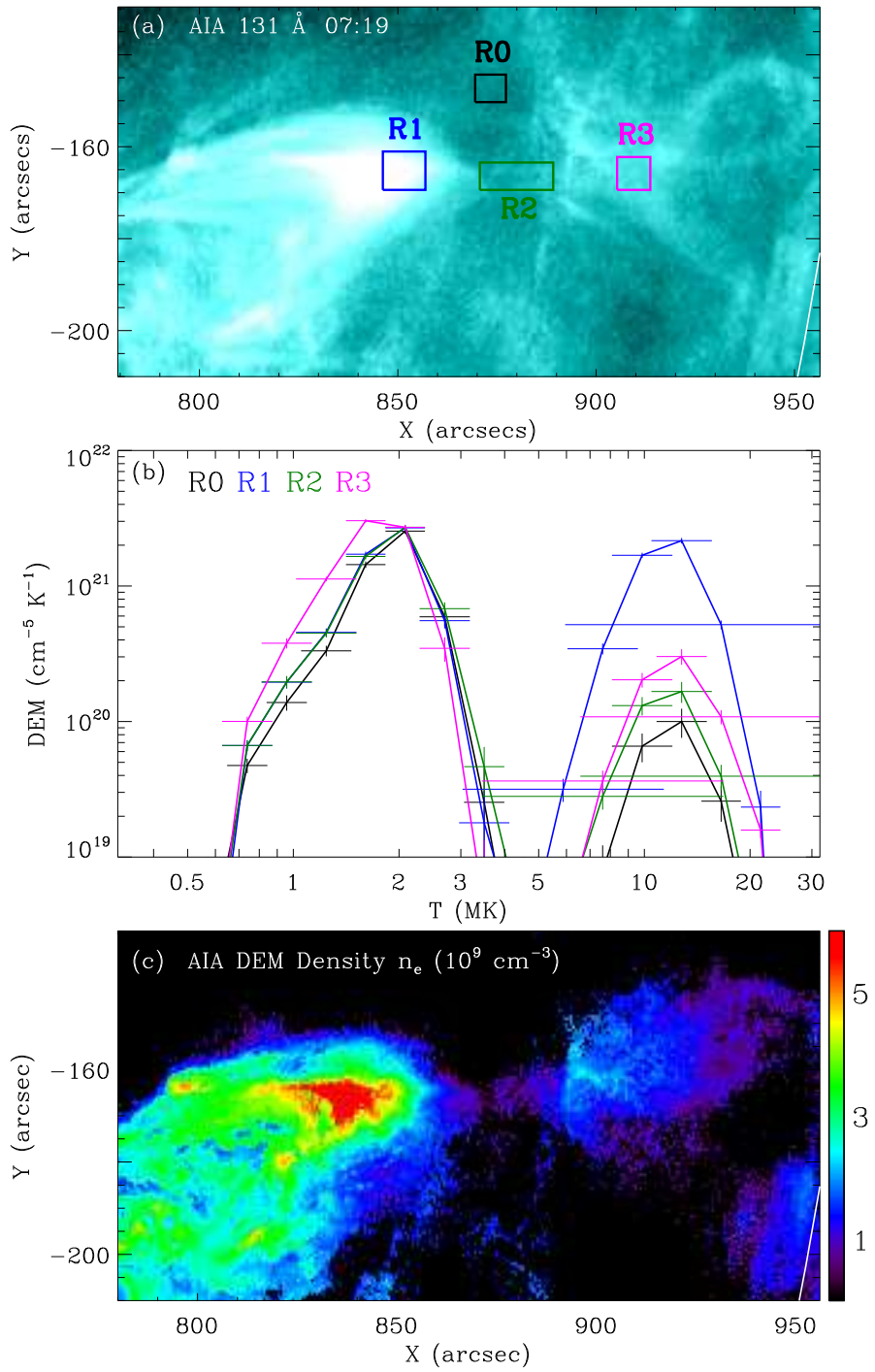

FIG. 5.- (a) Four regions, R0 - R3, are chosen to study DEM profiles, as displayed in (b). (c): Density map estimated from the DEM method with SDO/AIA images, at 07:19 UT.

mation temperature of Fe XxIV (O'Dwyer et al. 2010). Thus, the presence of any hotter plasma would not be detected.

By assuming that the depth of the CSLS, D, is comparable to $A_{\mathrm{cs}}{ }^{1 / 2}$, and that the plasma is uniformly distributed along its depth, a lower limit to the electron density can be estimated with $n_{e}=\sqrt{\mathrm{EM} / D}$, where EM is the emission measure. The derived density map is shown in Figure 5(c). The estimated densities are $3.0-$ $6.8 \times 10^{9} \mathrm{~cm}^{-3}$ at the flare top, $\sim 0.8 \times 10^{9} \mathrm{~cm}^{-3}$ at the CSLS, and $\sim 1.6 \times 10^{9} \mathrm{~cm}^{-3}$ at the CUF.

The magnetic reconnection rate, as denoted by the inflow Alfvén Mach number $M_{\mathrm{A}}=V_{\text {in }} / V_{\mathrm{A}} \approx V_{\text {in }} / V_{\text {out }}$, is evaluated in two ways. 1) By using the observed velocities of the inward $\left(V_{\text {in }} \sim 10 \mathrm{~km} \mathrm{~s}^{-1}\right)$ and outward ( $V_{\text {out }}$ $\sim 200 \mathrm{~km} \mathrm{~s}^{-1}$ ) flows, yielding $M_{\mathrm{A}} \approx 0.05$. 2) Using the temperature of the flaring regions ranging from $7-16$ MK and the assumption that the magnetic field energy is equally transformed into kinetic and thermal energy
(Ko et al. 2003), the Alfvén speed $V_{\mathrm{A}} \approx 198\left(\frac{T}{1 \mathrm{MK}}\right)^{1 / 2}$ $\mathrm{km} \mathrm{s}^{-1}$ (Liu et al. 2011) is $524-792 \mathrm{~km} \mathrm{~s}^{-1}$. With this value of $V_{\mathrm{A}}$, the reconnection rate is $0.01-0.02$. The relatively slower outflows, compared with the Alfvén speed, are probably caused by drag on these flows (Savage \& McKenzie 2011).

\section{DISCUSSION}

In this article, we study the formation and evolution of a CSLS, associated with loop-loop interactions, by using joint multi-wavelength observations with high spatial and temporal resolutions from SDO/AIA, STEREOA/EUVI and Hinode/XRT.

This study provides rare observational evidence and features for loop-loop interaction. 1) Several signatures of ongoing reconnection appear in a single event, such as converging motion, downward shrinking loops and the upward ejecting loops, blob-like structures, and a CSLS connecting the flare loop top and the CUF. 2) The reconstructed topology of two groups of interacting loops (Figure 1) indicate they are noncoplanar and involve kinked loops in the north, revealing the 3D aspects of this solar flare, and 3) The whole process involves the interactions between the kinked loops in the north with both long and short loops in the south. The appearance of the CSLS is probably a result of these interactions.

Our study reveals several important physical properties of the CSLS in loop-loop interaction. First, the length of the CSLS appeared to grow slowly with an average speed of $\sim 11 \mathrm{~km} \mathrm{~s}^{-1}$. The extension of the current sheet is expected during solar flares, and can grow as fast as a few hundred $\mathrm{km} \mathrm{s}^{-1}$ in the wake of an erupting CME (Forbes \& Lin 2000; Savage et al. 2010). The low growth speed here might be related to a different scenario, i.e. loop-loop interaction. Second, the observed erupting SHL, which initiated along with the downward shrinking loops, and appeared intermittently with an average velocity of $\sim 150 \mathrm{~km} \mathrm{~s}^{-1}$, might be related to the outward flows/blobs in current sheet (e.g., Shen et al. 2011). Third, the emission in AIA $193 \AA$ adjacent to the CSLS first increased, before its steep reduction in the late stage of the flare. This reduction in the emission is suggestive of the coronal dimmings frequently reported in association with CMEs (e.g., Sterling \& Hudson 1997; Zarro et al. 1999). In the present case, the dimmings are reported to result from a loop-loop interaction without evident detection of a CME. The relationship between the earlier increase in emission and the onset of the flare, if any, needs to be investigated in future studies.

The authors would like to thank the referees for many valuable comments that helped improve this article. We thank Wei Liu for helpful discussion. R.T.J.M. and C.Z. are funded by NSF-CAREER 1255024. D.A. acknowledges support by the NSF SHINE grant AGS-1061899. R.L. acknowledges the Thousand Young Talents Programme of China, and NSFC 41474151. We also thank SDO, STEREO, Hinode, SOHO and GOES for the data support. 


\section{REFERENCES}

Antonucci, E., Gabriel, A., Acton, L., et al. 1982, Sol. Phys., 78, 107

Battaglia, M., \& Kontar, E. P. 2012, ApJ, 760, 142

Ciaravella, A. \& Raymond, J. 2008, ApJ, 686, 1372

Fan, Y., \& Gibson, S. 2007, ApJ, 668, 1232

Forbes, T., \& Lin, J. 2000, J. Atmos. Sol.-Terr. Phys., 62, 1499

Furth, H. P., Killeen, J., \& Rosenbluth, M. N. 1963, Phys. Fluids, 6,459

Golub, L., DeLuca, E., Austin, G., et al. 2007, Sol. Phys., 243, 63

Hannah, I. G., \& Kontar, E. P. 2012, A\&A, 539, A146

Kaiser, M. L., Kucera, T. A., Davila, J. M., et al. 2008, Space Sci. Rev., 136, 5

Ko, Y. K., Raymond, J. C., Lin, J., et al. 2003, ApJ, 594, 1068

Kopp, R. A., \& Pneuman, G. W. 1976, Sol. Phys., 50, 85

Kosugi, T., Matsuzaki, K., Sakao, T., et al. 2007, Sol. Phys., 243, 3

Lemen, J. R., Title, A. M., Akin, D. J., et al. 2012, Sol. Phys., 275,17

Lin, J., Li, J., Forbes, T., et al. 2007, ApJ, 658, L123

Lin, J., Li, J., Ko, Y.-K., \& Raymond, J. 2009, ApJ, 693, 1666 Lin, J., Murphy, N. A., Shen, C., et al. 2015, Space Sci. Rev., 194,

Liu, R., Lee, J., Wang, T., et al. 2010, ApJ, 723, L28

Liu, R., Wang, T.-J., Lee, J., et al. 2011, Res. Astron. Astrophys., 11,1209

Liu, W., Chen, Q., \& Petrosian, V. 2013, ApJ, 767, 168

Loureiro, N., Schekochihin, A., \& Cowley, S. 2007, Phys. Plasmas, 14, 0703

Masuda, S., Kosugi, T., Hara, H., \& Ogawara, Y. 1994, Nature, 371,495

McKenzie, D. E.. \& Hudson, H. S. 1999, ApJ, 519, L93

O'Dwyer, B., Del Zanna, G., Mason, H. E., Weber, M. A., \& Tripathi, D. 2010, A\&A, 521
Pesnell, W. D., Thompson, B. J., \& Chamberlin, P. C. 2012 , Sol. Phys., 275, 3

Reeves, K. K., Linker, J. A., Mikić, Z., \& Forbes, T. G. 2010, ApJ, 721, 1547

Savage, S. L., \& McKenzie, D. E. 2011, ApJ, 730, 98

Savage, S. L., McKenzie, D. E., Reeves, K. K., Forbes, T. G., \& Longcope, D. W. 2010, ApJ, 722, 329

Schmelz, J., Kashyap, V., Saar, S., et al. 2009, ApJ, 704, 863

Schou, J., Scherrer, P., Bush, R., et al. 2012, Sol. Phys., 275, 229

Shen, C., Lin, J., \& Murphy, N. A. 2011, ApJ, 737, 14

Shibata, K., \& Tanuma, S. 2001, Earth Planets and Space, 53, 473

Sterling, A. C., \& Hudson, H. S. 1997, ApJ, 491, L55

Su, Y., Veronig, A. M., Holman, G. D., et al. 2013, Nat. Phys., 9, 489

Sui, L. H. \& Holman, G. D. 2003, ApJ, 596, L251

Sun, J., Cheng, X., Ding, M., et al. 2015, Nat. Commun., 6

Švestka, Z. F., Fontenla, J. M., Machado, M. E., et al. 1987, Sol. Phys., 108, 237

Takasao, S., Asai, A., Isobe, H., \& Shibata, K. 2012, ApJ, 745, L6

Thompson, W., Kliem, B., \& Török, T. 2012, Sol. Phys., 276, 241

Tian, H., Li, G., Reeves, K. K., et al. 2014, ApJ, 797, L14

Tsuneta, S., Hara, H. Shimizu, T., et al. 1992, PASJ, 44, L63

Wang, T., Sui, L., \& Qiu, J. 2007, ApJ, 661, L207

Webb, D., Burkepile, J., Forbes, T., \& Riley, P. 2003,

J. Geophys. Res.: Space Physics (1978-2012), 108

Wuelser, J. P., Lemen, J. R., Tarbell, T. D., et al. 2004, in Proc. SPIE, Vol. 5171, 111

Yokoyama, T., Akita, K., Morimoto, T., Inoue, K., \& Newmark, J. 2001, ApJ, 546, L69

Yokoyama, T., \& Shibata, K. 2001, ApJ, 549, 1160

Zarro, D. M., Sterling, A. C., Thompson, B. J., Hudson, H. S., \& Nitta, N. 1999, ApJ, 520, L139 